\title{
7. RADIO OBSERVATIONS OF X-RAY SOURCES
}

\author{
L. L. E. BRAES and G. K. MILEY \\ Leiden Observatory, The Netherlands
}

\begin{abstract}
Observational work on radio emission from galactic X-ray sources is reviewed. Some problems associated with the detection of these weak radio sources are mentioned and our present knowledge of radio identifications is summarized. The radio properties of the most studied objects are discussed in detail.
\end{abstract}

\section{Introduction}

The complementary nature of different branches of astronomy has rarely been more apparent than in the study of galactic $\mathrm{X}$-ray sources. Here we shall review the radio work on these sources, excluding objects identified with supernova remnants. Also we concentrate on the observational aspects, leaving the theoretical implications to Dr Hjellming (this volume, p. 98).

\section{Observational Techniques}

The first thing to bear in mind is the weakness of the radio emission we are dealing with. For example, the radio flux from Sco X-1, the brightest object in the X-ray sky, is more than four orders of magnitude weaker than that of Cas A, the brightest radio source. In this flux range, present single-dish radio telescopes are not noise limited, but are chiefly troubled by their inability to separate the many confusing sources in their beam. Instruments which use interferometric techniques to synthesize large apertures combine high sensitivity with high resolving power, and it is therefore no coincidence that almost all the observations we shall describe were made with these aperture-synthesis arrays.

A typical aperture-synthesis observation at wavelength $\lambda$ (see, e.g., Swenson, 1969) consists of measuring the complex fringe visibility outputs of $n$ interferometers with baselines increasing in length by a factor $d$ out to a maximum of $D=n d$. If a radio source is continually tracked over a range of hour angles, the Earth's rotation provides a two-dimensional array of projected baselines. A Fourier transform of the visibility data then yields a map of the radio brightness distribution with an east-west angular resolution $\approx \lambda / D$, over an area of sky determined by the beam of the primary antennas.

We shall be concerned mainly with contributions made by the National Radio Astronomy Observatory's (NRAO) three-element interferometer at Green Bank, U.S.A., operating at frequencies of 2695 and $8085 \mathrm{MHz}$, and the synthesis radio telescope at Westerbork (WSRT), the Netherlands, operating at $1415 \mathrm{MHz}$. Photographs of these arrays are shown in Figures 1 and 2. For a description of the characteristics of the instruments we refer to Hogg et al. (1969), Baars and Hooghoudt (1972), and Casse and Muller (1972); the relevant instrumental parameters are listed in Table I.

Because of its longer maximum baseline and higher observing frequencies, the 


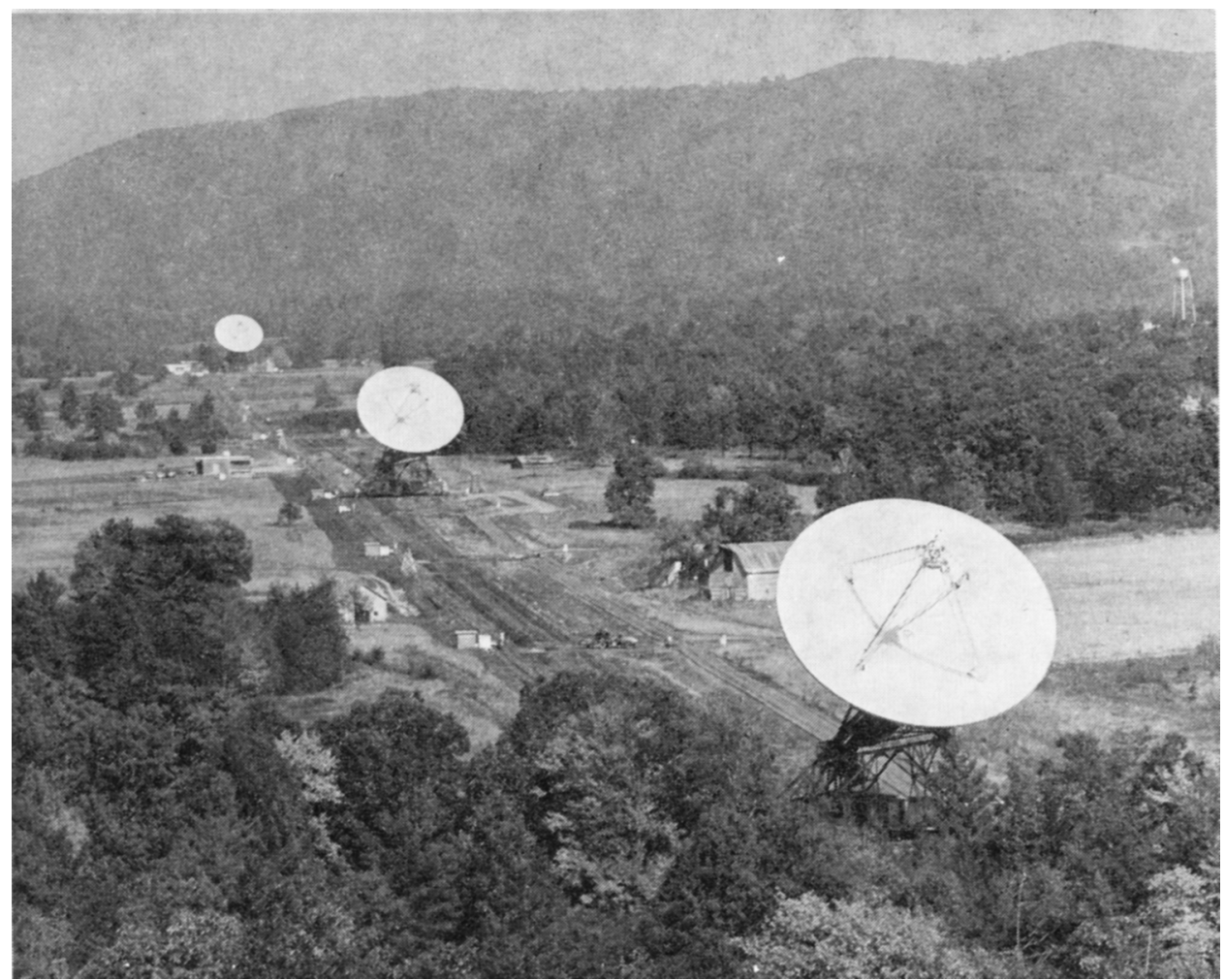

Fig. 1. The NRAO three-element interferometer.

\section{TABLE I}

Instrumental parameters of the NRAO and Westerbork synthesis telescopes

\begin{tabular}{|c|c|c|}
\hline & $\begin{array}{l}\text { NRAO } \\
\text { interferometer }\end{array}$ & WSRT \\
\hline Number of telescope elements & 3 & 12 \\
\hline Diameter of elements (m) & 25 & 25 \\
\hline Number of simultaneous baselines & 3 & 20 \\
\hline Maximum length (m) & 2700 & 1600 \\
\hline Orientation (azimuth in degrees) & 242 & 90 \\
\hline Operating frequencies $(\mathrm{MHz})$ & $8085 \quad 2695$ & 1415 \\
\hline wavelengths (cm) & 11.1 & 21.2 \\
\hline Bandwidth (MHz) & $2 \times 30 \quad 2 \times 30$ & $1 \times 4$ \\
\hline Resolution in right ascension $\left(\mathrm{s}^{\prime \prime}\right)$ & $3-8$ & 22 \\
\hline Sensitivity $\left(10^{-29} \mathrm{~W} \mathrm{~m}^{-2} \mathrm{~Hz}^{-1}\right)$ & 4 & 4 \\
\hline Half-power beamwidth (degrees) & 0.1 & 0.6 \\
\hline
\end{tabular}

a 3 rms noise after $12 \mathrm{hr}$ observation. 




Fig. 2. The Westerbork synthesis radio telescope (Copyright 'Aerophoto Eelde'). 
resolving power of the NRAO interferometer is superior to that of the WSRT. The broader bandwidth and lower-noise front ends of the NRAO instrument compensate in sensitivity for the fewer telescope elements.

A problem inherent in using the aperture-synthesis technique is that of confusion by diffraction grating rings. The response of a synthesis array is usually described in terms of a synthesized beam, i.e. the calculated response of the instrument to a point source observed with the same baseline coverage. In general, this beam will have diffraction grating rings due to the discrete nature of the baseline coverage, and these rings will surround the source at intervals whose separation on the synthesis map is inversely proportional to $d$. For many complex regions the limiting factor is not the sensitivity or the resolution, but the magnitude of confusion from grating rings. Here the WSRT has two immediate advantages. First, the large number of simultaneous baselines results in much fewer rings. Secondly, because of the east-west orientation of the telescope elements the rings are well defined ellipses, rather than complicated spiral patterns as produced by the skew baseline of the NRAO interferometer.

The highly variable nature of the radio emission from $\mathrm{X}$-ray sources also poses somewhat of a problem because a typical measurement is a synthesis of several hours observation. A variation in the flux of a source during this period causes a smearing of the response on the synthesis map. The variability may be analysed in small time intervals, but a limitation on the time resolution is, of course, set by the signal to noise ratio.

\section{Radio Identification of X-Ray Sources}

Location of a radio source within current X-ray error boxes is not sufficient for a secure identification, for the probability of a chance coincidence is in general not negligible. It is only when a source also exhibits unusually large and rapid variations that an identification is regarded as definite. This additional criterion is imposed because of the unique variability behaviour of the radio object coincident with the Sco X-1 X-ray star.

So far, the X-ray sky has been searched in only a preliminary way at radio wavelengths. The availability of aperture-synthesis instruments has restricted searches to declinations north of $\sim-40^{\circ}$, and only the brightest sources with positions known to better than a few minutes of arc have been observed.

Table II summarizes the present status of the results. It contains all the published NRAO and Westerbork identification data together with some unpublished results of Westerbork measurements. Note that the quoted flux densities do not refer to simultaneous observations.

Of the 14 sources in the table, five now have well established radio counterparts. Two others have radio sources within the X-ray error boxes, but these have not yet been found to be variable. Upper limits are available for the radio emission of the remaining seven objects.

We should also mention the result of a recent radio search for Her X-1 (2U $1705+$ +34) conducted by the MIT group; with the NRAO interferometer four sources 


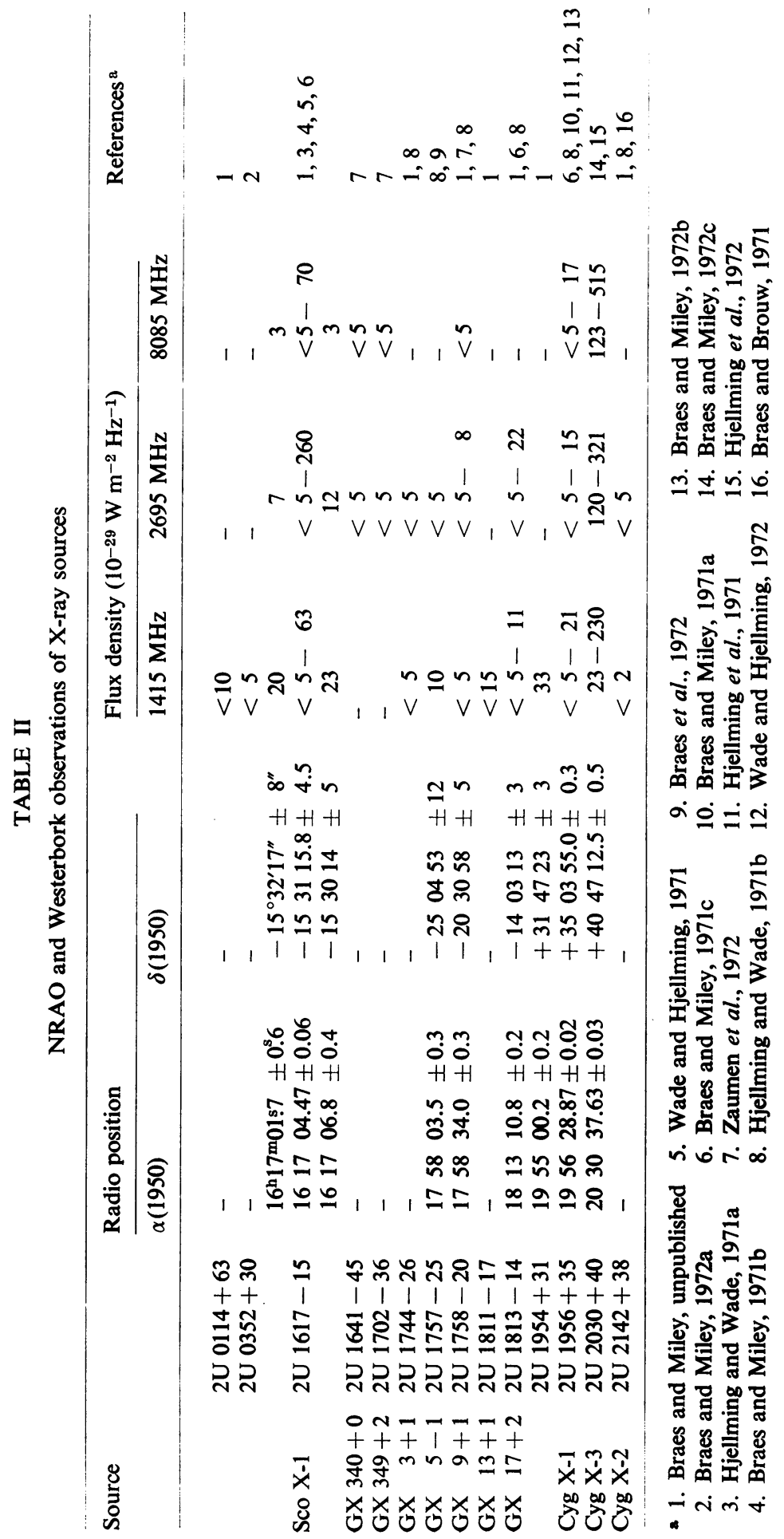


were detected which they consider as candidates for the radio counterpart of this pulsing X-ray source (Doxsey et al., 1972).

\section{Remarks on Individual Objects}

In this section we shall successively discuss in some detail the results for Sco X-1, the Cygnus sources, and the sources in the galactic centre region.

\section{A. SCORPIUS X-1}

Sco X-1 was the first X-ray star to be detected at radio wavelengths (Andrew and Purton, 1968). Variation in its radio flux was first reported by Ables (1969).

Perhaps the most intriguing feature is its triple radio structure, first revealed by NRAO observations (Hjellming and Wade, 1971a) and confirmed later by Westerbork measurements (Braes and Miley, 1971b). Sco X-1 appears remarkably like a miniature quasar, with a central highly variable component coinciding in position to within $1^{\prime \prime}$ with the X-ray star, and two companion sources located symmetrically on either side of it, 1.18 to the north-east and 1:24 to the south-west.

The outer components have non-thermal spectra, with spectral indices $\sim-1$ above $1415 \mathrm{MHz}$. From a comparison with the $408 \mathrm{MHz}$ result obtained by Jauncey (1971),

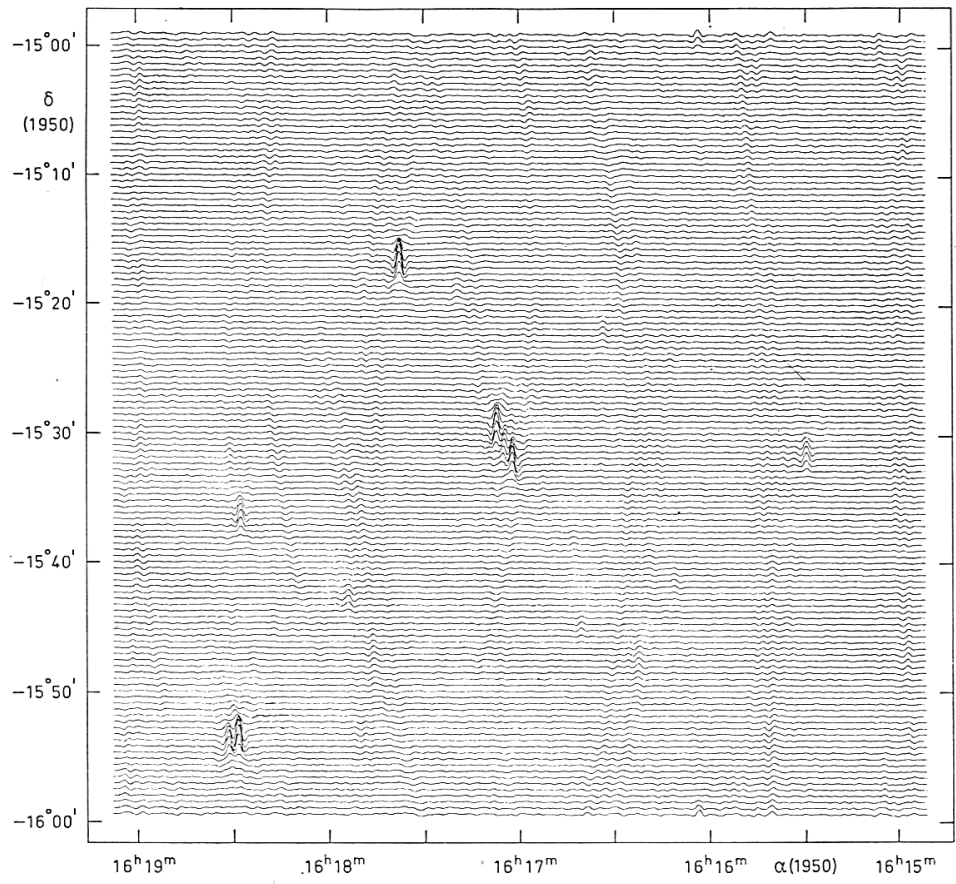

Fig. 3. Westerbork $1415 \mathrm{MHz}$ map of the Sco X-1 field, presented as a series of intensity profiles in right ascension. The map combines $99 \mathrm{hr}$ of observations to give an rms noise of about $0.5 \times 10^{-29} \mathrm{~W} \mathrm{~m}^{-2} \mathrm{~Hz}^{-1}$. 
at least one appears to become optically thick below this frequency. Because these properties are typical of normal extragalactic sources, the question arises whether the companions are physically related to the X-ray star, or whether they are just background objects. As Sco X-1 is situated in an uncrowded region well away from the galactic plane, the probability of a chance coincidence is small. This can be seen from Figure 3, showing the $1415 \mathrm{MHz}$ map of the surrounding $1^{\circ} \times 1^{\circ}$ region. Additional evidence for a relationship comes from recent, unpublished, NRAO and Westerbork results indicating that the north-east companion is variable.

If the two sources are relativistic plasmoids ejected from the star, it is conceivable that their proper motions should be measurable. Assuming a distance of $300 \mathrm{pc}$, a transverse velocity of $0.01 \mathrm{c}$ would result in an apparent expansion of $2^{\prime \prime}$ per year. Such a measurement is difficult, however, because of distortion by sidelobes from the variable central component and by the elongated beam due to the low declination. Present results are inconclusive.

The central component has been studied extensively (Wade and Hjellming, 1971; Braes and Miley, 1971c). Its spectrum is non-thermal with an index typically $\sim-0.5$ between 2695 and $8085 \mathrm{MHz}$. The few simultaneous NRAO and Westerbork measurements suggest that it becomes optically thick between 1415 and $2695 \mathrm{MHz}$.

Figure 4 illustrates how the activity of the central source can vary from day to day; in $24 \mathrm{hr}$ its mean flux density increased from 7 to $44 \times 10^{-29} \mathrm{~W} \mathrm{~m}^{-2} \mathrm{~Hz}^{-1}$. It also varies erratically on a time scale of minutes, but searches for pulsar-like behaviour have so far proved negative (see, e.g., Taylor et al., 1972). Lampton et al. (1971) failed

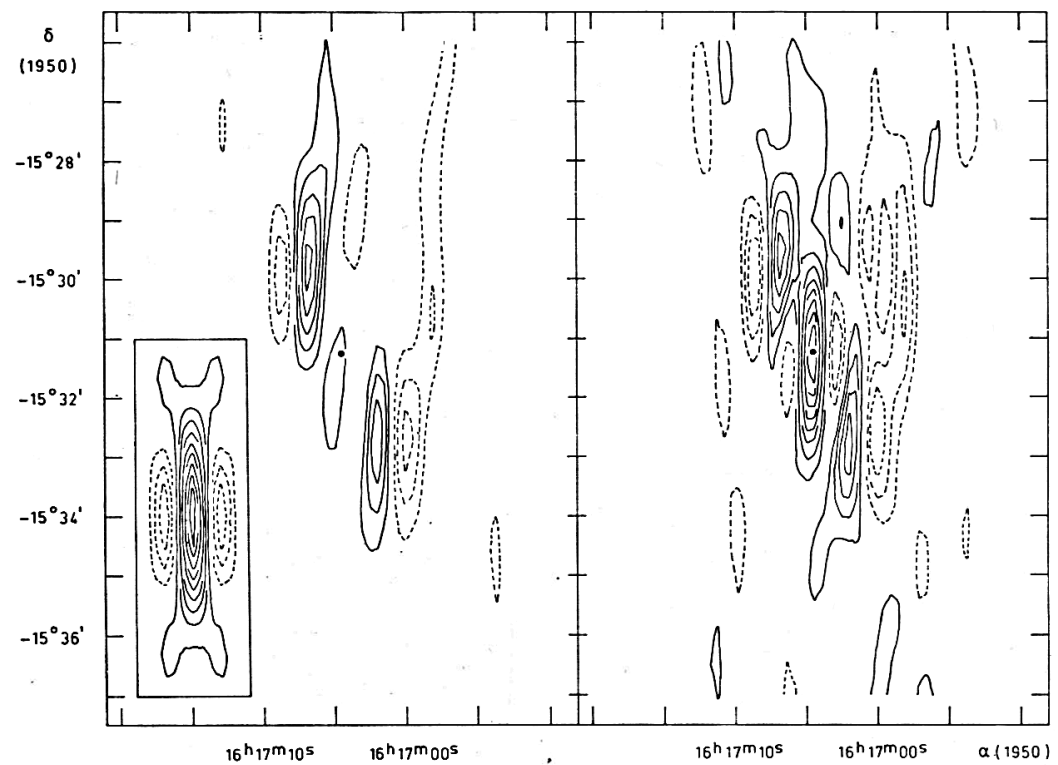

Fig. 4. $1415 \mathrm{MHz}$ maps of Sco X-1 on March 29 (left) and March 30, 1971 (right). The contours give levels of equal brightness, the dashed ones representing negative intensities. For comparison, the antenna pattern is shown inset. The black dot marks the position of the X-ray star. 
to find any correlation between the optical and radio variations, and Dr Hiltner (this volume, p. 74) just pointed out that though considerably more data now exist, evidence for a direct correlation is still lacking.

\section{B. CYGNUS X-1}

Like that of Sco X-1, the radio counterpart of Cyg X-1 is strongly variable, but in a very different way. It appeared suddenly within a three-weeks period, just over a year ago (Braes and Miley, 1971a; Hjellming and Wade, 1971b). Previously, the Cyg X-1 field had been observed three times at NRAO and once at Westerbork without success. Figure 5 shows that apart from a slight decrease in flux after its initial

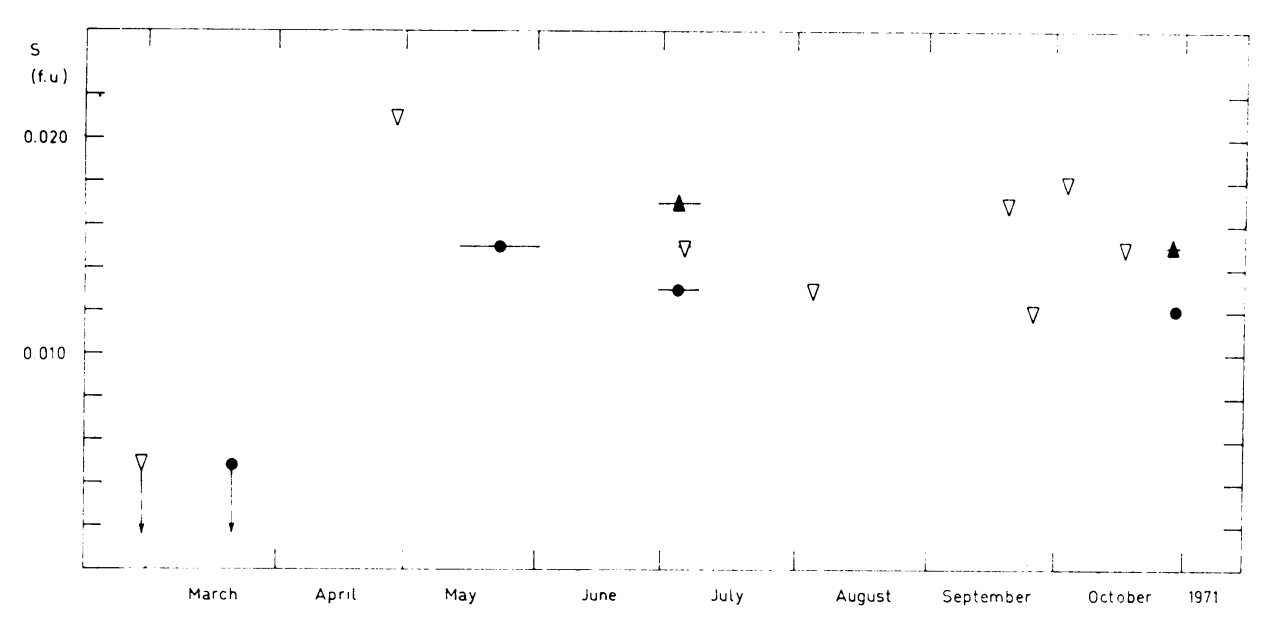

Fig. 5. The flux density of Cyg X-1 as a function of time. Included are Westerbork measurements at $1415 \mathrm{MHz}(\nabla)$ and NRAO data at $2695(O)$ and $8085 \mathrm{MHz}(\Delta)$. Where measurements have been combined this is indicated by horizontal bars. The rms error in an individual point is about $0.004 \mathrm{fu}=4 \times 10^{-29} \mathrm{~W} \mathrm{~m}^{-2} \mathrm{~Hz}^{-1}$.

appearance, there is little evidence for subsequent radio variability. Also no pulsarlike behaviour has been found.

A compilation of data at four frequencies from Westerbork, NRAO, and Algonquin Radio Observatory, indicates that the Cyg X-1 radio source has a peculiar spectrum characteristic of the active extragalactic sources, with a minimum between 1415 and $8085 \mathrm{MHz}$ (Hjellming et al., 1971).

Figure 6 shows how the optical identification of Cyg X-1 was made possible by the interplay of $\mathrm{X}$-ray and radio techniques. The detection of a radio counterpart narrowed down the positional uncertainty and led to the identification with the B0 Ib star HDE 226868. Subsequent refinements of the early radio positions shown in Figure 6 left no doubt regarding the positional coincidence with this spectroscopic binary (Wade and Hjellming, 1972; Braes and Miley, 1972b). 


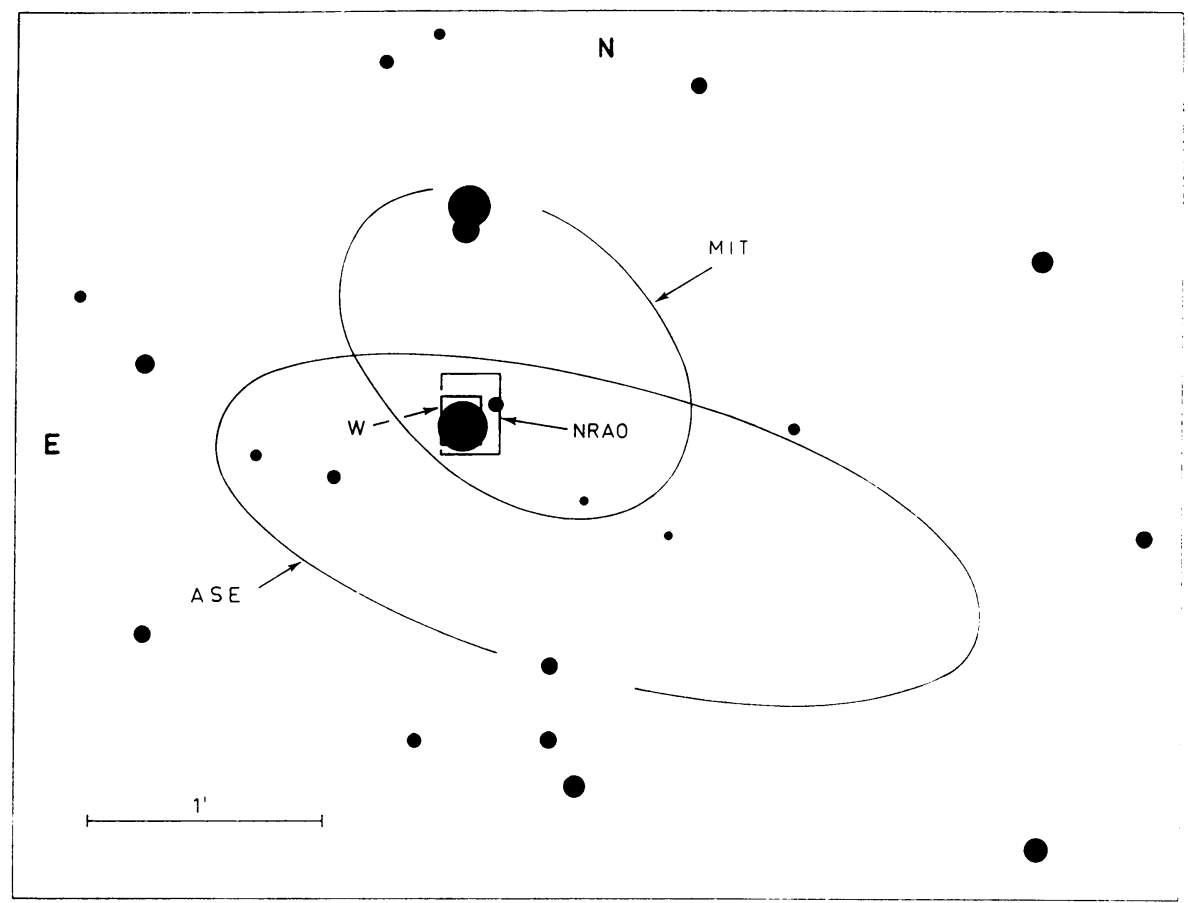

Fig. 6. Identification of Cyg X-1 with HDE 226868. Superimposed on the star field are the UHURU (private communication) and MIT (Rappaport et al., 1971) X-ray error ellipses and the (3 rms) radio error boxes.

\section{CYGNUS $\mathrm{X}-2$}

No radio emission has yet been detected from the star generally assumed to be the optical counterpart of Cyg X-2. There is a point source nearby, but earlier (Westerbork) indications of variability have not been confirmed (Braes and Brouw, 1971; Hjellming and Wade, 1971b).

\section{CYGNUS X-3}

Recently, Westerbork observations have led to the detection of a highly variable radio source near the X-ray position of Cyg X-3 (Braes and Miley, 1972c). Over a period of eight months its $1415 \mathrm{MHz}$ flux density was observed to vary by an order of magnitude, and also on a time scale of hours it appears to vary appreciably. As Dr Hjellming will relate (this volume, p. 98), this extreme variability has been confirmed by subsequent NRAO measurements.

The Cyg X-3 radio source is remarkably strong; the ratio of its radio to X-ray emission is about 50 times greater than for Cyg X-1 and exceeds that for Sco X-1 by more than two orders of magnitude.

Cyg X-3 is located in the crowded Cyg X radio complex, and Figure 7 shows the many sources in the field. This map is a complete synthesis resulting from a combination 


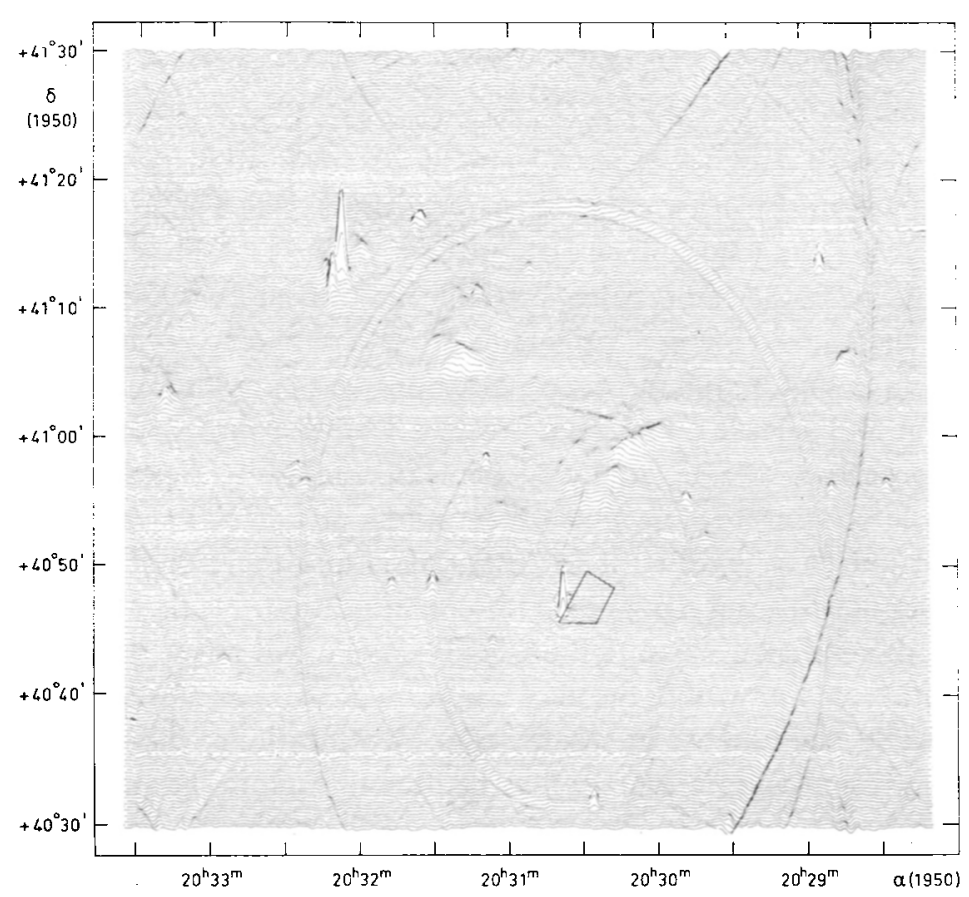

Fig. 7. Westerbork complete-synthesis map of the $1415 \mathrm{MHz}$ brightness distribution in a $1^{\circ} \times 1^{\circ}$ region surrounding Cyg X-3. The quadrilateral marks the $90 \%$ confidence limits of the 2 ASE X-ray position for Cyg X-3. Noise in this figure amounts to about $0.7 \times 10^{-29} \mathrm{~W} \mathrm{~m}^{-2} \mathrm{~Hz}^{-1}$.

of data from four separate days. The variable source stands out due to the surrounding grating rings, caused by the changes in flux level during the period covered by the observations.

An attempt at optical identification has met with no success, but this is not surprising since the whole region is obscured by the Great Cygnus Rift.

\section{E. GALACTIC CENTRE SOURCES}

If these objects are indeed at the distance of the galactic centre, one would expect their apparent radio strengths to be relatively low and difficult to measure. Radio identification problems are aggravated by the low declination and increased confusion.

At present, only GX $9+1$ and GX $17+2$ have been positively detected (Hjellming and Wade, 1971b; Braes and Miley, 1971c; Zaumen et al., 1972); both sources exhibit short-term variability of the Sco X-1 type. There is also a radio source located within the GX 5-1 X-ray error box (Braes et al., 1972), but definite identification must await the detection of variability. As can be seen from Table II, NRAO and Westerbork searches at the positions of GX $3+1,13+1,340+0$, and $349+2$ have been negative. Cruder upper limits at $4.6 \mathrm{~cm}$ were previously set by Andrew et al. (1970).

No optical counterparts have yet been found for any of the galactic centre sources. 


\section{Concluding Remarks}

From this review it is clear that the radio observations are as yet in their infancy; we only have limited measurements of some of the strongest X-ray sources. Even so, we can already distinguish two classes, namely those that exhibit short-term variability (e.g. Sco X-1) and those that do not (Cyg X-1). The failure to find any direct correlations between the radio behaviour and the optical and $\mathrm{X}$-ray characteristics implies that the radio emission originates from a different region of the objects.

It is important that simultaneous observations over a wide range of frequencies are carried out to illucidate the spectral properties of the sources, and because of their unpredictable behaviour and large dispersion in luminosity repeated monitoring is needed. It is interesting to note that observations of Cyg X-1 over an eight-month period would not have revealed it to be variable, and therefore failure to measure variability does not rule out a possible radio identification. Neither does the complete absence of radio emission mean that an X-ray source will never be detectable.

We can anticipate that within the next few years as more and better X-ray positions become available, the number of secure radio identifications will increase manyfold. Also, intercontinental interferometry may be used to explore the structural variations of some of the stronger objects. Because several of their radio properties are so typical of extragalactic sources and because they are so relatively nearby, it is tempting to speculate that radio observations of galactic X-ray sources may contribute to our knowledge of violent events taking place far outside our galaxy.

Note added in proof: An unprecedented radio outburst was observed in Cygnus X-3 during early September 1972 (IAU Circ. No. 2440). Its spectral behaviour resembled that of an expanding synchrotron source with a maximum flux density of $22 \times 10^{-26}$ $\mathrm{W} \mathrm{m} \mathrm{m}^{-2} \mathrm{~Hz}^{-1}$ at $10630 \mathrm{MHz}$. This giant flare will be discussed in Nature during October 1972.

\section{References}

Ables, J. G.: 1969, Astrophys. J. 155, L27.

Andrew, B. H. and Purton, C. R.: 1968, Nature 218, 855.

Andrew, B. H., Purton, C. R., Rappaport, S., Bradt, H., and Schnopper, H. W.: 1970, Astrophys. J. 161, L173.

Baars, J. W. M. and Hooghoudt, B. G.: 1972, Astron. Astrophys., in preparation.

Braes, L. L. E. and Brouw, W. N.: 1971, Astron. Astrophys. 12, 320.

Braes, L. L. E. and Miley, G. K.: 1971a, Nature 232, 246.

Braes, L. L. E. and Miley, G. K.: 1971b, Astron. Astrophys. 14, 160.

Braes, L. L. E. and Miley, G. K.: 1971c, Veröff. Remeis-Sternw. Bamberg IX, No. 100, 173 (IAU

Coll., No. 15, 'New Directions and New Frontiers in Variable Star Research').

Braes, L. L. E. and Miley, G. K.: 1972a, Nature 235, 273.

Braes, L. L. E. and Miley, G. K.: 197.2b, Nature Phys. Sci. 235, 147.

Braes, L. L. E. and Miley, G. K.: 1972c, Nature 237, 506.

Braes, L. L. E., Miley, G. K., and Schoenmaker, A. A.: 1972, Nature 236, 392.

Casse, J. L. and Muller, C. A.: 1972, Astron. Astrophys., in preparation.

Doxsey, R., Murthy, G. T., Rappaport, S., Zaumen, W., and Spencer, J.: 1972, Astrophys. J. 176, L15. 
Hjellming, R. M. and Wade, C. M.: 1971a, Astrophys. J. 164, L1.

Hjellming, R. M. and Wade, C. M.: 1971b, Astrophys. J. 168, L21.

Hjellming, R. M., Wade, C. M., Hughes, V. A., and Woodsworth, A.: 1971, Nature 234, 138.

Hjellming, R. M., Hermann, M., and Webster, E.: 1972, Nature 237, 507.

Hogg, D. E., Macdonald, G. H., Conway, R. G., and Wade, C. M.: 1969, Astron. J. 74, 1206.

Jauncey, D. L.: 1971, Nature Phys. Sci. 230, 200.

Lampton, M., Bowyer, S., Welch, J., and Grasdalen, G.: 1971, Astrophys. J. 164, L61.

Rappaport, S., Zaumen, W., and Doxsey, R.: 1971, Astrophys. J. 168, L17.

Swenson, G. W.: 1969, Ann. Rev. Astron. Astrophys. 7, 353.

Taylor, J. H., Huguenin, G. R., and Hirsch, R. M.: 1972, Astrophys. J. 172, L17.

Wade, C. M. and Hjellming, R. M.: 1971, Astrophys. J. 170, 523.

Wade, C. M. and Hjellming, R. M.: 1972, Nature 235, 271.

Zaumen, W., Murthy, G. T., Rappaport, S., Hjellming, R. M., and Wade, C. M.: 1972, Nature 235, 378. 